\title{
15-year incidence of new-onset diabetic ketoacidosis in children with type 1 diabetes from a regional paediatric setting (Auckland, New Zealand)
}

Craig Jefferies ${ }^{*}$, Samuel Cutfield ${ }^{2}$, José GB Derraik ${ }^{2}$, Jignal Bhagvandas ${ }^{1}$, Benjamin B Albert ${ }^{2}$, Paul L Hofman², Alistair J Gunn ${ }^{3}$, Wayne S Cutfield ${ }^{2,4}$

From 8th APPES Biennial Scientific Meeting

Darwin, Australia. 29 October - 1 November 2014

\section{Aims}

This study aimed to assess the incidence of new-onset diabetic ketoacidosis (DKA) incidence over a 15-year period in children with type 1 diabetes (T1DM) from our regional paediatric diabetes centre in Auckland, New Zealand.

\section{Methods}

We performed a retrospective review of all patients $<15$ years of age diagnosed with T1DM from our unselected complete regional cohort for the years 1999 to 2013. Children were included if they had type 1 diabetes as defined by clinical presentation and/or DKA and/or presence of pre-type 1 diabetes autoantibodies. DKA was classified into Mild ( $\mathrm{pH}<7.3$ bicarbonate $<15$ ), Mod $(\mathrm{pH}<7.2$ bicarbonate $<10)$, and severe $(\mathrm{pH}<7.1$ bicarbonate $<5$ ), according to the ISPAD guidelines.

\section{Results}

For the 15-year period, there were 731 children $<15$ years with new-onset T1DM, 343 (47\%) males, there were 195 (26.7\%) cases of new-onset DKA: 51 (26\%) severe, 52 (27\%) moderate, and 92 (47\%) with mild DKA. Average age at diagnosis was 8.6 years. The annual incidence of DKA was variable, ranging from $18 \%$ to $37 \%$. The overall incidence of new-onset DKA did not differ over the study interval ( $\mathrm{p}=0.78$ ). The likelihood of being in DKA at onset of T1DM was unaffected by age, sex, ethnicity, or socioeconomic status. Amongst those in DKA, New Zealand
European ethnicity $(\mathrm{p}=0.038)$ and female gender $(\mathrm{p}=0.056)$ were each associated with increasing DKA severity at presentation.

\section{Conclusions}

There has been a stable but persistent level of New-onset DKA over the 15-year period studied in our regional paediatric population. Action must be taken to improve awareness of T1DM and in doing so, reduce the incidence of new-onset DKA.

\section{Authors' details \\ ${ }^{1}$ Starship Children's Hospital, Auckland, New Zealand. 'Liggins Institute, University of Auckland, Auckland, New Zealand. ${ }^{3}$ Department of Physiology, University of Auckland, Auckland, New Zealand. ${ }^{4}$ Gravida National centre for growth and development, Auckland, New Zealand.}

Published: 28 April 2015

doi:10.1186/1687-9856-2015-S1-P3

Cite this article as: Jefferies et al: 15-year incidence of new-onset diabetic ketoacidosis in children with type 1 diabetes from a regional paediatric setting (Auckland, New Zealand). International Journal of Pediatric Endocrinology 2015 2015(Suppl 1):P3.

'Starship Children's Hospital, Auckland, New Zealand

Full list of author information is available at the end of the article 